\title{
Wireless Sensor Network Achieved by Automatic Positioning System Node
}

\author{
Yang Zhongguo and Cai Tianfang \\ College of Mechanical and Electrical Engineering, Zaozhuang institute, 277160, \\ China \\ 13589610269@163com
}

\begin{abstract}
In application of wireless sensor network, the position information of sensor node is crucial within life cycle in the whole network. In order to design algorithm of node positioning with high-efficiency, high-precision and low-consumption, this paper puts forward using the encryption algorithm of space control node originally applied in field of engineering measurement, by using SDI algorithm (space and distance intersection algorithm) as three-dimensional position algorithm used in system to achieve threedimensional automatic positioning calculation of distributed nodes. Test proves that this kind of algorithm has better positioning nature and higher precision; it is used as threedimensional positioning because it has good engineering application value.
\end{abstract}

Keywords: Wireless sensor network, automatic positioning of node, beacon node, positioning system, positioning algorithm

\section{Introduction}

WSNS is the self-organization network [1] composed by large number of sensor nodes with sensing capacity, calculation capacity and communication capacity, as the new information acquisition and procession technology, it has universal application prospect [2] in field related to positioning such as environment monitoring, target tracking, intelligent traffic, intrusion detection etc. In theses applications, obtaining monitoring information mostly needs to know the position information of sensor node in WSNS, otherwise, the collected information is meaningless or invalid [3], so it is very important to obtain the node information of sensor. The sensor nodes of WSNS is usually distributed at random with large-scale, due to its restriction conditions such as limited capacity and weak reliance, limited communication distance of wireless module, which puts forward higher requirements to achieve technology and algorithms related to self-positioning, it is improper to use routine GPS positioning or site engineering measurement. The selfpositioning system applied in wireless sensor network node often requires characteristics such as self-organization, robustness, energy high-efficiency and distributed computation etc.

This paper designs one self-positioning system of three-dimensional space node based on portable beacon node, it also introduces into space and distance intersection algorithm as three-dimensional position algorithm of it. It makes evaluation and analysis on SDI algorithm as well as overall capability of positioning system by simulated test.

\section{Self-positioning of Wireless Sensor Network}

Node positioning of WSNS means the relative position or absolute coordinates of unknown node in network. Node positioning means setting certain number of anchor nodes in network, it is also regarded as beacon node, and these anchor nodes can obtain information of self-position by carrying positioning equipment or pre- 
calibration, the other common sensor nodes beyond of anchor nodes are unknown node. Generally speaking, the scale of anchor node in network node is small; it is mainly used as the reference node of positioning unknown node, which means unknown node confirms its position by position information of anchor node and other related information. In the WSNS indicated in Figure 1, B represents anchor node and $\mathrm{U}$ represents unknown node. $\mathrm{U}$ node communicates with $\mathrm{B}$ node by means of multi-hop transmission and obtains the distance, angle, network connectivity or relevance information with $\mathrm{B}$ node by using certain distance or non-distance technology as well as certain algorithm, finally it uses positioning algorithm to calculate the position of unknown node.

In wireless sensor network, the measurement technologies mostly used on distance or angle between nodes is as follows: RSSI, TOA, TDOA and AOA, RSSI and TDOK are frequently used. Because of the limited resources of WSNS, largescale network and distribution randomness, the capacity of positioning system and algorithm will directly affect its applicability. How to evaluate position algorithm is one problem worth doing profound research. The standard of algorithm judgment is as follows: low consumption, robustness, distribution, self-organization, anchor node density, node density, positioning precision etc, supposing that WSNS has N sensor nodes, of which the number of anchor node is $M$ and number of unknown node is $\mathrm{N}-\mathrm{M}$. The actual coordinates of sensor node $\mathrm{Xi}$ is $\mathrm{xi}$, of which, $\mathrm{i}=1 \ldots \mathrm{N}$.

The estimated coordinates of positioning algorithm for unknown node $\mathrm{Xi}$ is ${ }_{-}, \quad \mathrm{i}=$ $1 \ldots \mathrm{N}-\mathrm{M}$, its average positioning deviation is defined as follows: of which $\|\cdot\| 2$ means the Euclid distance between 2 nodes. Usually, the smaller the average positioning deviation, the better of capability of positioning algorithm.

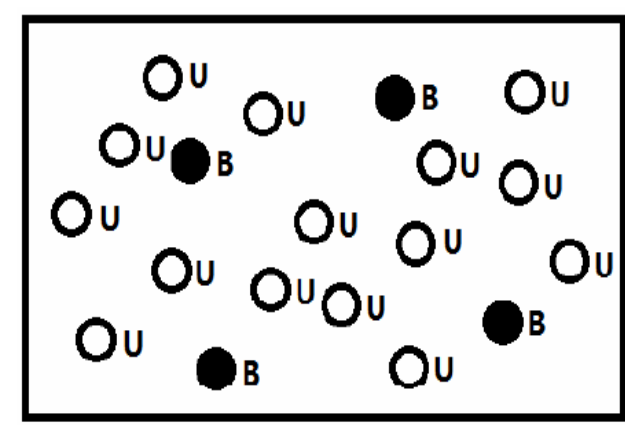

Figure 1. The Anchor Node and Unknown Node in WSNS Network

$$
\text { error }=\frac{\sum_{i=1}^{N-M}\left\|\bar{x}_{i}-x_{i}\right\|_{2}}{N_{-} M}
$$

The typical positioning algorithm such as three-edge positioning algorithm, centroid positioning algorithm, SPA relative positioning algorithm, convex programming algorithm, APS positioning algorithm, DV-distance algorithm, Euclidean positioning algorithm, DV-coordinate positioning algorithm, MDS-MAP positioning algorithm [4] etc. For these algorithms, all have their limitations, the existing nodes self-positioning system and algorithm all has respective characteristic and applicable range, there is no absolutely best. From the overall, the algorithm system including some recycling refinement steps proposed in recent years, such as DV-coordinate positioning algorithm, MDS-MAP positioning algorithm etc, which fully displays characteristics of wireless sensor network, that it realizes the tasks that single node can't complete by using coordinated work among nodes. This kind of algorithm system emphasizes on the estimated calculation of node position in the 
early stage, it can seek cycling refinement according to the precision threshold iterative preset by users, and it can also use the whole refinement stage as the optional state according to application demand. But the unknown node must directly connect with the beacon node, many algorithms and positioning capabilities have larger improvement space for the defects such as higher requirement for beacon node density, reliance of positioning precision on network arrangement condition, low convergence speed etc. Problem of node self-positioning is still one technical difficulty for the research of wireless sensor network; it is also one of the key points.

\section{Research on Three-Dimensional Space Self-Positioning of Wireless Sensor Network Node}

\subsection{Design of Node Self-Positioning System}

In this stage, we can use the multi-hop transmission of wireless sensor network to realize information sharing between nodes, coordinately to achieve distance estimated calculation between single node and node group. It is indicated in Figure 2 , supposing that aircraft flying at low space carrying one signal transmitting device circling over the monitoring area, it can be regarded as the mobile beacon node in the positioning system. Mobile beacon node is supposed having long-term and unlimited energy supply to support signal transmission, it can use GPS or other portable positioning device to determine its position, so that it causes much more deviation for mapping technology. Therefore, the exist of obstacle has obvious effect on positioning precision of positioning algorithm(such as three-edge positioning algorithm, triangle positioning algorithm and maximum likelihood estimation etc).

Three-dimensional positioning process of nodes can be divided into 2 steps:

(1) Each unknown node receives the signal from portable beacon node, it will use this to measure distance with the beacon node at the signal transmission position, and obtain the information and space and position of beacon node at that time. Supposing that beacon node carries with the carries with transmissible super wide band and can simultaneously launch two mutual intersected UWB pulse wave as distance measurement training data as well as other data information, unknown node receives UWB signal and obtains data information, it also uses TOA technology to measure distance.

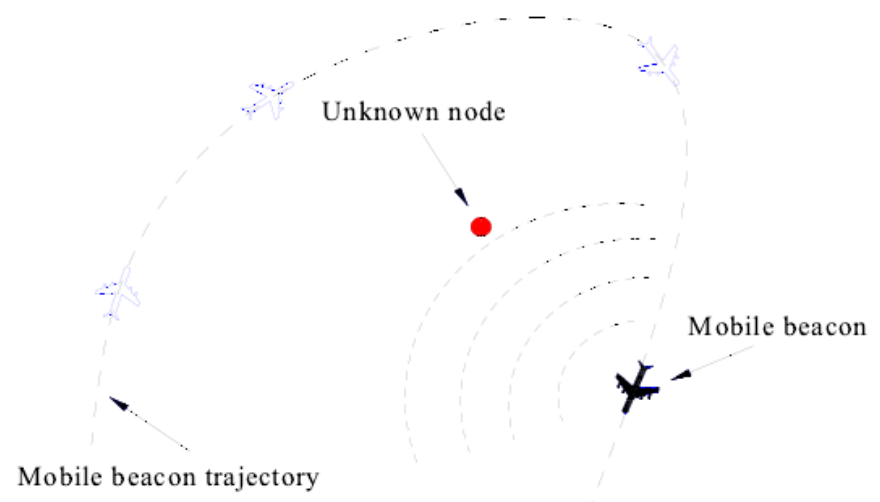

Figure 2. Using Portable Beacon Node to Assist Unknown Nodes Sketch 
(2) Each known node can obtain the three-dimensional position through certain position calculation algorithm according to one group of measurement information of beacon node located in different space and position in Step 1.

\subsection{Space and Distance Intersection Algorithm}

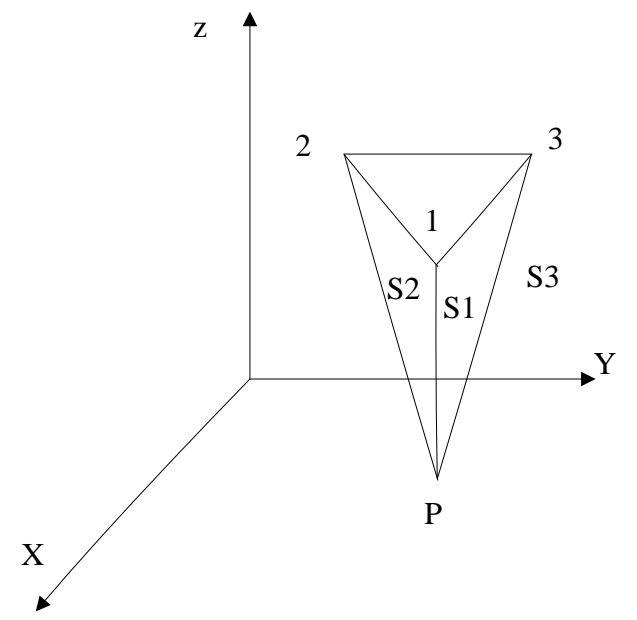

Figure 3. SDI Algorithm Diagram

SDI algorithm is the usually used in the encryption control point in 3D space used in field of engineering measurement, and it is retrograded as one flexible, effective and practical three-dimensional position algorithm [8]. It is indicated in Figure 3, 1, 2, 3 are three control points, their coordinates are already known, they are respectively (x1, x2, $\mathrm{x} 3),(\mathrm{y} 1, \mathrm{y} 2, \mathrm{y} 3),(\mathrm{z} 1, \mathrm{z} 2, \mathrm{z} 3), \mathrm{P}$ is the point to be located. We will set up observation station in point $\mathrm{P}$ to respectively measure the space distance $\mathrm{S} 1, \mathrm{~S} 2$ and $\mathrm{S} 3$ between $\mathrm{P}$ and 1,2 and 3, according to this, we can adopt SDI algorithm to calculate the 3D space coordinates $(\mathrm{x}, \mathrm{y}, \mathrm{z})$ of point $\mathrm{P}$.

Firstly, we can construct one three pyramid body, its vertex is $\mathrm{P}, 1,2$ and 3, according to vector algebra theory, the volume of three pyramid body is calculated by the following formula: of which

, $\cos \alpha_{1}, \cos \beta_{1}, \cos \gamma_{1}$ is the cosine of $\mathrm{P}$ direction.

$$
\begin{aligned}
& V=\frac{1}{6}\left|\begin{array}{lll}
x-x_{1} & \mathrm{y}-\mathrm{y}_{1} & \mathrm{z}-\mathrm{z}_{1} \\
x-x_{2} & \mathrm{y}-\mathrm{y}_{2} & \mathrm{z}-\mathrm{z}_{2} \\
x-x_{3} & \mathrm{y}-\mathrm{y}_{3} & \mathrm{z}-\mathrm{z}_{3}
\end{array}\right|= \\
& \frac{1}{6} S 1 S 2 S 3\left|\begin{array}{llll}
\cos \alpha_{1} & \cos \beta_{1} & \cos \gamma_{1} \\
\cos \alpha_{2} & \cos \beta_{2} & \cos \gamma_{2} \\
\cos \alpha_{3} & \cos \beta_{3} & \cos \gamma_{3}
\end{array}\right| \\
& =\frac{1}{6} S 1 S 2 S 3 \vec{A}
\end{aligned}
$$

Then the value of $\mathrm{A}$ of determinant can be indicated as follows:

$$
A=\sqrt{\begin{array}{l}
\sin ^{2} \varphi_{12}+\sin ^{2} \varphi_{13}+\sin ^{2} \varphi_{23} \\
+2 \cos \varphi_{12} \cos \varphi_{13} \cos \varphi_{23}-2
\end{array}}
$$

$\Phi 12, \Phi 13$ and $\Phi 23$ are respectively the intersection angles of vector P1 and P2, P1 and P3, P2 and P3. Their sine and cosine value can be got by the known side length of $\Delta 1 \mathrm{P} 2$, 
$\Delta 1 \mathrm{P} 3$ and $\Delta 2 \mathrm{P} 3$, using sine principle and sine, cosine reduction formula. We define the following vector as follows:

$$
\begin{aligned}
& \left(x_{2}-x_{1}, \mathrm{y}_{2}-y_{1}, \mathrm{z}_{2}-z_{1}\right)=\left(X_{2}, Y_{2} \cdot H_{2}\right), \\
& \left(x_{3}-x_{1}, \mathrm{y}_{3}-y_{1}, \mathrm{z}_{3}-z_{1}\right)=\left(X_{3}, Y_{3} \cdot H_{3}\right), \\
& \left(x-x_{1}, \mathrm{y}-y_{1}, \mathrm{z}-z_{1}\right)=(X, Y, H)
\end{aligned}
$$

According to the above calculation formula of internal volume and mixed volume, we can list the following equations:

$$
\left\{\begin{array}{l}
\left|\begin{array}{ll}
\mathrm{Y}_{2} & \mathrm{H}_{2} \\
\mathrm{Y}_{3} & \mathrm{H}_{3} \\
\mathrm{X}_{2} X+Y_{2} Y+H_{2} H=K_{2} \\
\mathrm{X}_{3} X+\mathrm{H}_{3} Y+\mathrm{H}_{3} H=\mathrm{X}_{3}
\end{array}\right| Y+\left|\begin{array}{ll}
\mathrm{Y}_{2} & \mathrm{X}_{2} \\
\mathrm{Y}_{3} & \mathrm{X}_{3}
\end{array}\right| H=K_{1}
\end{array}\right.
$$

By calculation, then we get the coordinates of point to be determined as follows:

$$
x=X+x_{1}, y=Y+y_{1}, z=Z+z_{1}
$$

That is to say, we use the space position of mobile beacon node of three non-collinear received by one node to make code according to the clockwise sequence on the level, then take the corresponding coordinates and space data into the above calculation formula.

\subsection{Analysis on Positioning Precision}

Because the measurement value of S1, S2 and S3 has deviation, so it results in that calculation position of point $\mathrm{P}$ by using SDI algorithm also has deviation. This chapter uses the deviation of point $\mathrm{P}$ to measure positioning precision of SDI algorithm and gives deviation and some relative data of points, the detailed algorithm is as follows: for formula (2) to make differential treatment and get formula (7), cxy is the indication of direction cosine, for the details please see reference [10].

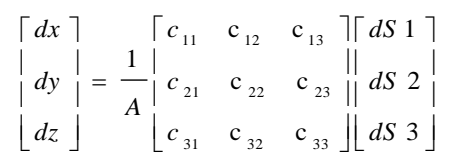

Because dS1, dS2 and dS3 are mutually independent, therefore, for the (7) application deviation propaganda principle, we can get the following deviation relation formula:

$$
\left\{\begin{array}{l}
m_{x}^{2}=1 / N\left(c_{11}^{2} m^{2}{ }_{s 1}+c_{12}^{2} m^{2}{ }_{s 2}+c_{13}^{2} m^{2}{ }_{s 3}\right) \\
m_{y}^{2}=1 / N\left(c_{21}^{2} m^{2}{ }_{s 1}+c_{22}^{2} m^{2}{ }_{s 2}+c_{23}^{2} m^{2}{ }_{s 3}\right) \\
m_{z}^{2}=1 / N\left(c_{31}^{2} m^{2}{ }_{s 1}+c_{32}^{2} m^{2}{ }_{s 2}+c_{33}^{2} m^{2}{ }_{s 3}\right)
\end{array}\right.
$$

Of which, mx 、 my and $\mathrm{mz}$ are respectively the deviation of three coordinate components in three-dimensional position of point to be determined, $\mathrm{mSi}(\mathrm{i}=1,2,3)$ is the deviation of space measurement of three sides.

Take (3) calculation into formula (8), it can get the precision estimated formula of three coordinate components in three-dimensional coordinates of point to be determined. According to positioning result of SDI algorithm, estimated formula [10] of level point precision and elevation point precision, it can get that level point precision and elevation point precision just related to the three controlled pitch (V1, V2, V3) by intersection angle and each measurement side, this brings convenience for practical application. 


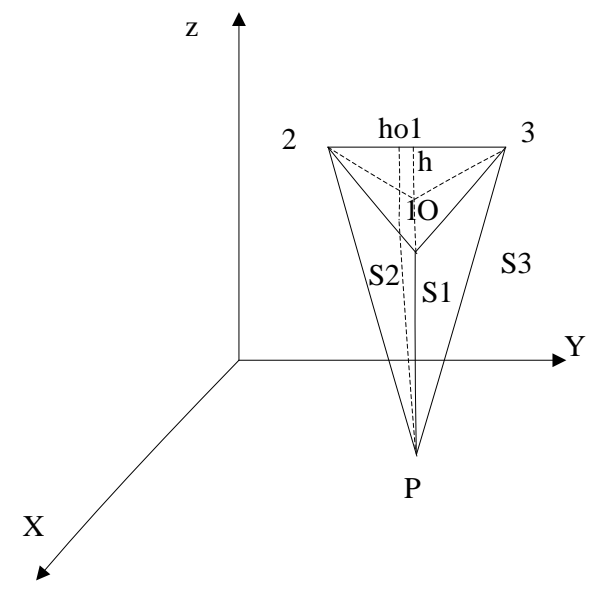

Figure 4. Parameter Diagram of Space Rendezvous Scheme

Indicated by Figure 4, setting level composed by three control point vertical elevation $\mathrm{H}$ direction, it can get the following:

$$
S V_{i}=\frac{h}{S_{i}}
$$

Takes it into the estimated formula of level point precision and elevation point precision, it can get the following:

$$
m_{\text {平 }}^{2}=\frac{\mathrm{D}_{10}^{2}}{\mathrm{~h}_{1}^{2}} \frac{m_{s 1}^{2}}{\cos ^{2} V_{1}}+\frac{\mathrm{D}_{20}^{2}}{\mathrm{~h}_{2}^{2}} \frac{m_{s 2}^{2}}{\cos ^{2} V_{2}}+\frac{\mathrm{D}_{30}^{2}}{\mathrm{~h}_{3}^{2}} \frac{m_{s 3}^{2}}{\cos ^{3} V_{3}}
$$

We can see from the above that: except $\mathrm{m}$ level related to space measurement precision, it is mainly dependent on configuration composed by control points and $\mathrm{P}$ points of the triangle in the bottom projection position as well as edge pitch range, when it gives three control points, $\mathrm{m}$ is mainly dependent on the pitch of each measurement distance and projection value of slant-range.

Take the relation of elevation point precision $\mathrm{mH}$ and each measurement distance pitch $\mathrm{Vi}$ into the estimated formula of level point precision and elevation point precision, it can get the following:

$$
m_{H}^{2}=\frac{\mathrm{D}_{10}^{2}}{\mathrm{~h}_{1}^{2}} \frac{m_{S 1}^{2}}{\sin ^{2} V_{1}}+\frac{\mathrm{D}_{20}^{2}}{\mathrm{~h}_{2}^{2}} \frac{m_{S 2}^{2}}{\sin ^{2} V_{2}}+\frac{\mathrm{D}_{30}^{2}}{\mathrm{~h}_{3}^{2}} \frac{m_{S 3}^{2}}{\sin ^{3} V_{3}}
$$

From the above, we can see that $\mathrm{mH}$ except related to measurement distance precision, it is mainly dependent on the projection point $\mathrm{O}$ at bottom and the connection of control points, divide $\Delta 123$ into three square and pitch of each measurement distance, when it gives three control points, $\mathrm{mH}$ is mainly dependent on pitch of each measurement distance and projection value.

We can get the relations of 3D point precision MP with pitch Vi of each measurement distance by formula (10) and (11):

$$
\begin{aligned}
& M_{p}^{2}=m_{\text {平 }}^{2}+m_{H}^{2}=\frac{\mathrm{D}_{10}^{2}}{\mathrm{~h}_{1}^{2}} \frac{m_{S 1}^{2}}{\cos ^{2} V_{1}}+\frac{\mathrm{D}_{20}^{2}}{\mathrm{~h}_{2}^{2}} \frac{m_{S 2}^{2}}{\cos ^{2} V_{2}}+ \\
& \frac{\mathrm{D}_{30}^{2}}{\mathrm{~h}_{3}^{2}} \frac{m_{S 3}^{2}}{\cos ^{3} V_{3}}+\frac{\mathrm{D}_{10}^{2}}{\mathrm{~h}_{1}^{2}} \frac{m_{S 1}^{2}}{\sin ^{2} V_{1}}+ \\
& \frac{\mathrm{D}_{20}^{2}}{\mathrm{~h}_{2}^{2}} \frac{m_{S 2}^{2}}{\sin ^{2} V_{2}}+\frac{\mathrm{D}_{30}^{2}}{\mathrm{~h}_{3}^{2}} \frac{m_{S 3}^{2}}{\sin ^{3} V_{3}}
\end{aligned}
$$


The above formula is the relation repression of 3D point precision MP and pitch Vi of each measurement distance, obviously, compared with level point precision or relation of elevation point precision to $\mathrm{Vi}$, it is more complicated. When Vi becomes larger, level point precision decreases, but elevation point precision increases, on the contrast, when $\mathrm{Vi}$ decreases, elevation point precision decreases, but level point precision increases.

\section{Simulated Analysis}

As shown in Figure 5, we choose square area $(100 \times 100$ units $)$ as the experimentation area, and randomly distribute 225 nods on the above area; the elevation point of each node selects random value between 0 and 20. Supposing that mobile beacon node is hovering above the area, its flying height is located at the height of 50 units higher than the average elevation point of unknown node. Supposing the percentage of beacon node is $50 \%$ and the aircraft carrying with beacon node is hovering above the area, it is flying at the concentric circle path approximately covering the whole area on the level position, but it can adjust the different flying height according to regional surface undulation. Supposing that the communication radius of mobile beacon node is 100 units, this it can enable each node to at least receive 8 positioning signal. Supposing that measurement values obey the normal distribution and use actual truth value as average value, one hundred ratio of it is the standard deviation, the change range of standard ratio is set as 0 to 0.25 .

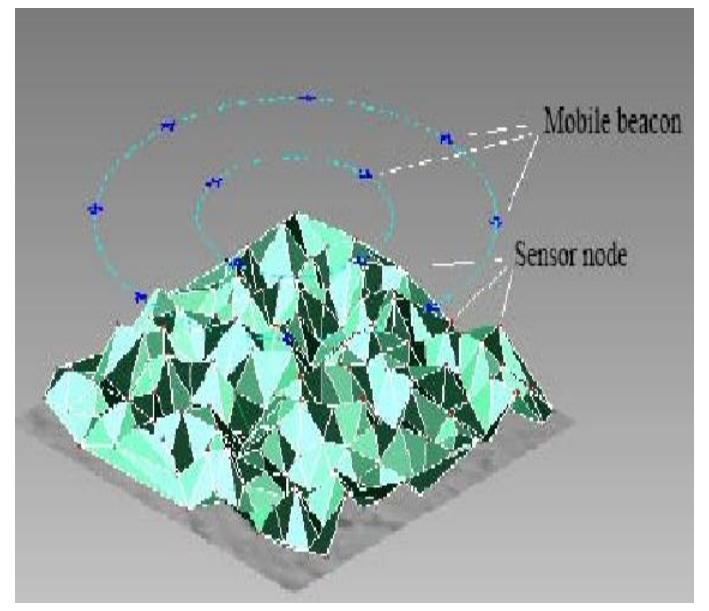

Figure 5. Scene Diagram of Simulated Test

In order to reliably measure the performance of algorithm, under deviation condition of certain measurement distance (using certain standard deviation to indicate), then correspondingly, carrying 100 times of self-positioning simulated test in the node of the whole range, afterwards, all the average point deviation by the effective positioning node after 100 times experiments is regarded as the positioning deviation by $3 \mathrm{D}$ position algorithm of node. 


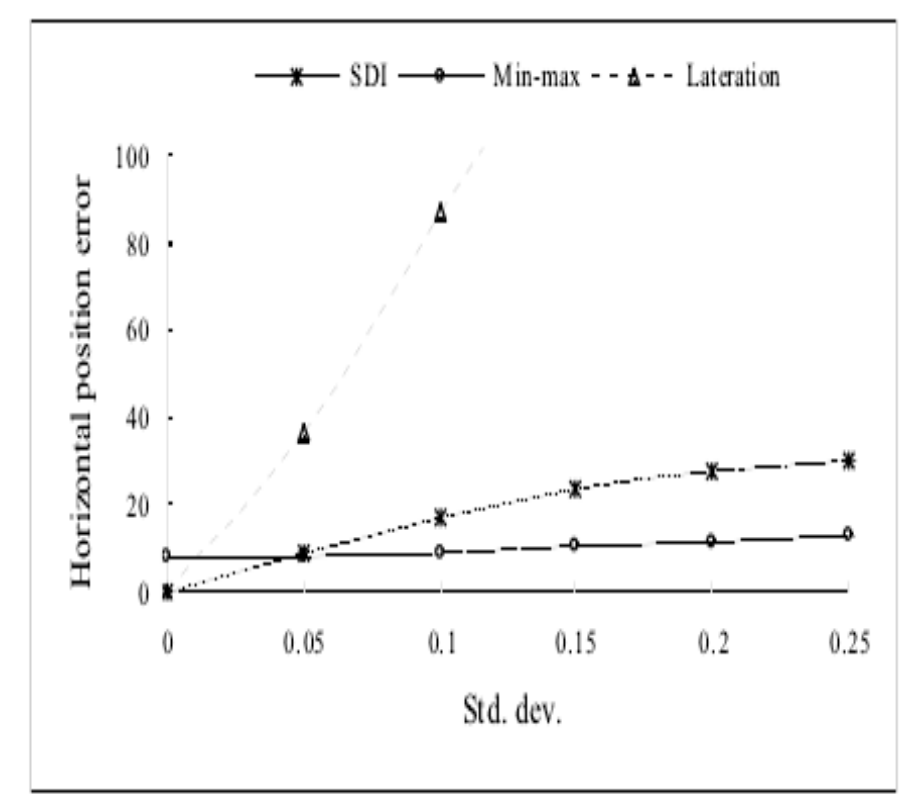

Figure 6. Level Position Deviation vs. Standard Deviation of Measurement Distance Deviation

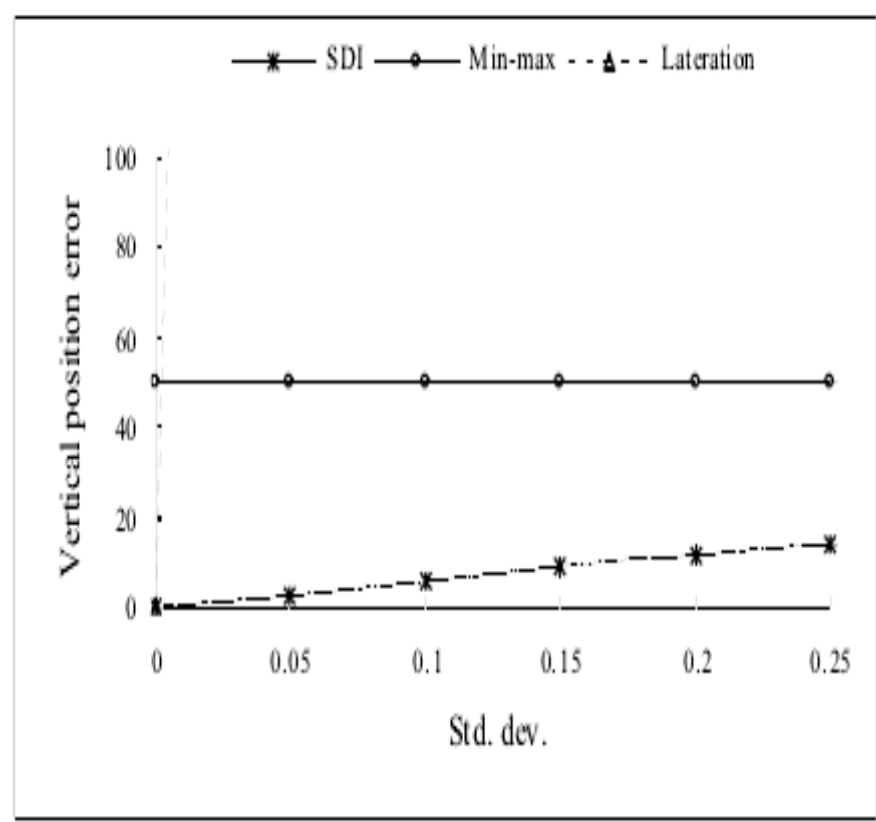

\section{Figure 7. Elevation Deviation vs. Standard Deviation of Measurement Distance Deviation}

Figure 6 and 7 are respectively indicated under different deviation conditions the performance of SDI, Lateration and Min-max in positioning height and elevation positioning precision. According to the curve in the figure, Lateration is the worst in three position algorithms, it is indicated that it is very sensitive to change of deviation of measurement distance(when the standard deviation of measurement distance larger than 0.1 , and positioning deviation even larger than 100, it cannot give reasonable positioning value). Min-max is not sensitive to deviation of measurement distance, so it keeps relatively stable positioning precision, including level positioning precision and elevation 
positioning precision. SID is also sensitive to deviation of measurement distance, when the measurement distance is relatively precise (ratio of standard deviation $\leq 0.05$ ), it indicates the level positioning precision superior to Min-max, and it keeps the maximum superiority on elevation point positioning precision under condition of measurement distance deviation. Compared with performance of SDI in level positioning precision and elevation positioning precision, we can see that positioning precision of SDI elevation point is superior to its level positioning precision.

Figure 8 indicates the positioning coverage of three kinds of position algorithms under different measurement distance deviation conditions, from the curve in the figure we can see that Min-max keeps high and stable coverage. When the deviation of measurement distance gradually increases, coverage positioning of SDI is gradually decreasing, when ratio of standard deviation larger than 0.3 , the coverage percent reduces to below $50 \%$. At this time judgment of procedure on unknown node cannot be positioned, so positioning coverage will decrease and it can avoid doing positioning calculation for node with large deviation on measurement distance.

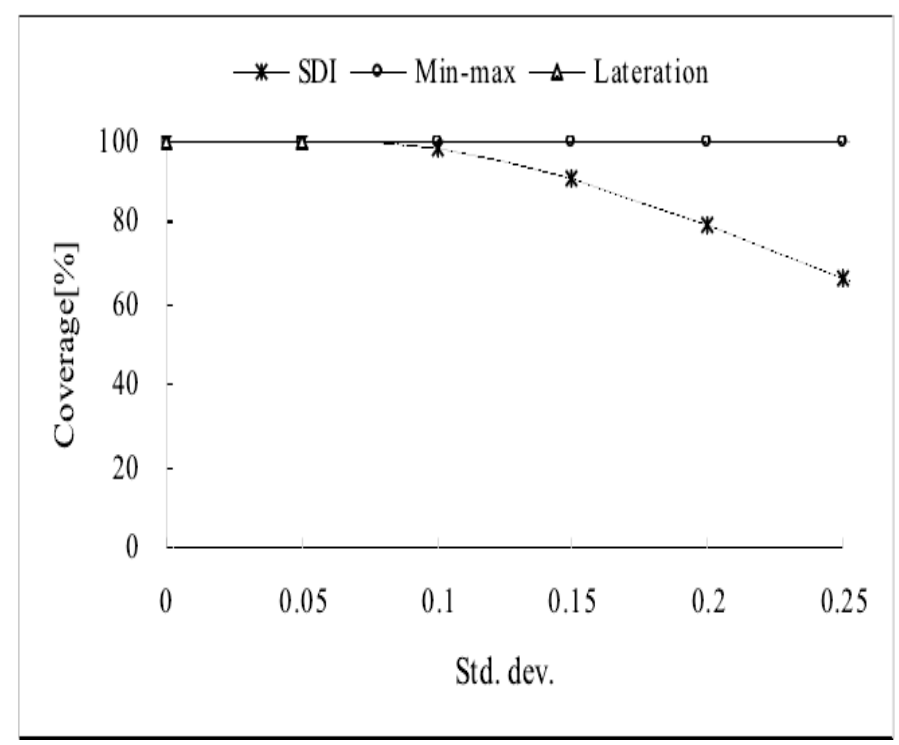

Figure 8. Elevation Deviation vs. Standard Deviation of Measurement Distance Deviation

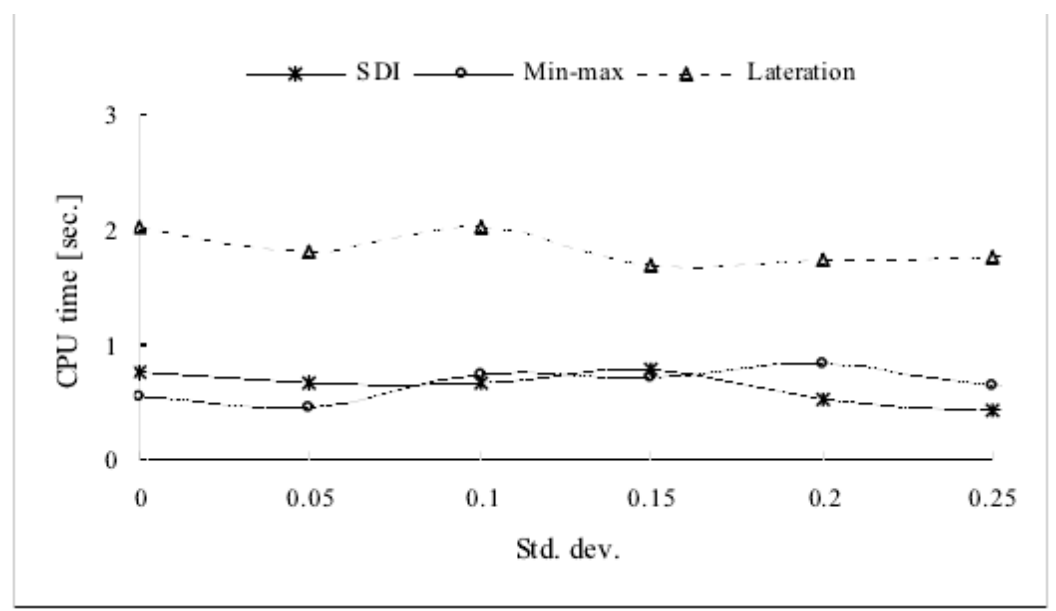

Figure 9. Elevation Deviation vs. Standard Deviation of Measurement Distance Deviation 
Figure 9 indicates the CPU consumption of three kinds of algorithms under different deviation conditions, from the curve in the figure, we can see that under the condition of the same positioning coverage, SID and Min-max algorithm almost have the same CPU consumption, while CPU consumption of Lateration is the biggest in three kinds of algorithms, it is several times of other two algorithms.

\section{Conclusions}

This paper puts forward one node three-dimensional self-positioning system based on empty carrier mobile beacon node, this system includes two basic positioning steps: space measurement distance and 3D self-positioning calculation. In the stage of space measurement distance, the unknown node in monitoring area is passively receives the UWB signal launched by empty-carrier mobile beacon node to obtain positioning information, and uses TOA measurement technology to measure the space distance between beacon node and the node position at that time, in the 3D self-positioning calculation stage, it proposes using space control point encryption algorithm originates from engineering measurement field, SDI algorithm to realize 3D position calculation of node.

For the proposed positioning system, because it adopts UWBTOA measurement technology, it can meet requirement of SDI algorithm for precision measurement, so positioning system can obtain better 3D positioning result in the whole. In addition, restricted by conditions, it can obtain relatively low measurement precision, according to the above performance analysis of each algorithm, taking SDI and Min-max combination used in the 3D self-positioning calculation of wireless network node is one optimized plan, the positioning result is composed by level coordinates by Min-max algorithm and elevation position coordinates by SDI algorithm, while SDI algorithm has coverage problem when the measurement precision is relatively low, it can increase signal coverage of mobile beacon or adjacent node of realizing 3D self-positioning for elevation of unknown node to implement elevation interpolation.

\section{Acknowledgements}

The authors would express their appreciation for the financial support of Shandong Natural Science Foundation, grant NO. ZR2011EL016. The authors also would express their thanks for Shandong Science and Technology Development Project on Safety in Production, grant NO. LAJK2013-183.

\section{References}

[1] S. Qinqin, "Self-positioning System and Algorithm Study on Wireless Sensor Network Node, (2009), Shanghai Jiao Tong University.

[2] J. K. Hart and K. Martinez, "Environmental Sensor Network: A revolution in the earth system science", Earth Science Reviews, vol. 78 nos. 3-4, (2006), pp. 177-191.

[3] C. Xunxue, Z. Zhan and W. Cheng, "Application Field and Design Technology of Wireless Sensor Network, National Defense Industrial Press, (2009).

[4] S. Kim, D. Pakzad, J. C. Demmel, et al., "Health monitoring of civil infrastructures using wireless sensor networks", Cambridge, MA, United states, (2007), pp. 254-263.

[5] C. T. Xuan, E. Kim and I. Koo, "An efficient RSS-based localization scheme with calibration in wireless sensor networks," IEICE Transactions on Communications, E91-B, no. 12, (2008), pp. 4013-4016.

[6] X. Lei, "Node Positioning Algorithm of Step Precision of One Kind of Wireless Sensor Network Appliance and Instrumentation Journal", vol. 29, no. 2, (2008), pp. 314-319.

[7] W. H. Liao, Y. C. Lee and S. P. Kedia, "Mobile anchor positioning for wireless sensor networks", Iet Communications, vol. 5, no. 7, (2011), pp. 914-92.

[8] W. R. Chang, H. T. Lin and Z. Z. Cheng, "CODA: A continuous object detection and tracking algorithm for wireless ad hoc sensor networks", 2008 5th IEEE Consumer Communications and Networking Conference, Las Vegas, NV, United states, (2008), pp. 168-174. 
[9] W. K. Lui, H. C. So H C and W. K. Maximum, "A Posteriori Approach to Time-of-Arrival based Localization in Non-Line-of-Sight Environment", IEEE Transactions on Vehicular Technology, vol. 59, no. 3, (2010), pp. 1517-1523.

[10] H. S. Ahn and W. Yu, "Environmental-Adaptive RSSI-Based Indoor Localization", IEEE Transactions on Automation Science and Engineering, vol. 6, no. 4, (2009), pp. 1545-5955.

[11] W. Fubao, S. Long and R. Fengyuan, "Self-positioning System and Algorithm of Wireless Sensor Network", Software Journal, vol. 16, no. 5, (2005), pp. 857-868.

[12] W. Shaohua and Z. Naitong, "Two-step Estimated Algorithm Based on UWB Wireless Sensor Network", Software Journal, vol. 18, no. 5, (2007), pp. 1164-1172.

[13] W. Fubao, "Self-positioning System and Algorithm of Wireless Sensor Network", Software Journal, vol. 16 , no. 5, (2005), pp. 857-868.

[14] R. Fengyuan, H. Haining and L. Chuang, "Wireless Sensor Network", Software Journal, 2003, vol. 14, no. 7, (2003), pp. 1282-1290.

[15] S. Long, "Range-free Self-positioning Mechanism and Algorithm of Wireless Sensor Network, Computer Engineering and Application, vol. 40, no. 23, (2004), pp. 127-130.

\section{Authors}

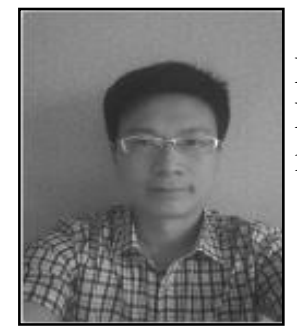

Yang Zhongguo, male, lecturer at College of Mechanical and Electrical Engineering, Zaozhuang institute, Dean of Mechanical and Electrical Engineering majors section, main research areas are mechanical and electrical automation, wireless sensor network etc.

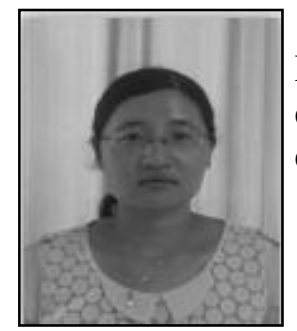

Cai Tianfang, female, lecturer at College of Mechanical and Electrical Engineering, Zaozhuang institute, Dean of Control engineering majors section, main research areas are mechanical and electrical automation, wireless sensor network etc. 
International Journal of Future Generation Communication and Networking Vol. 8, No. 2 (2015) 